\title{
Fugue and aphonia as first and foremost presentations of a case of psychosis
}

\author{
Christina Leotsakou ${ }^{1 *}$, Andreas Sardis ${ }^{2}$, Periklis Robotis ${ }^{1}$, Christos Labiris ${ }^{1}$, Maria Giovanoglou' ${ }^{1}$ Periklis Paterakis ${ }^{1}$ \\ From $1^{\text {st }}$ International Congress on Neurobiology and Clinical Psychopharmacology and European \\ Psychiatric Association Conference on Treatment Guidance \\ Thessaloniki, Greece. 19-22 November 2009
}

\section{Background}

Hysteria has experienced many changes in diagnostic classification and clinical manifestations. It has become infrequent due to cultural but also diagnostic changes. A change in which people communicate their distress has taken place. In the past, bodily experiences used to express emotions whereas nowadays a shift from bodily experiences to emotional -or better to say psychologicalexperiences has been proposed. "Unable to speak but can whisper", "Cannot hold arm but can hold it in place", "Glove and stocking pain or numbing": all these represent the patient's idea of anatomy and physiology, and were seen more often in the past. On the other hand hysterical behavior has for long been described in cases of endogenous psychoses.

\section{Materials and methods}

Case Report: A 48-year-old woman, married, mother of three children, was admitted to our hospital for aphonia for the last year, severe anxiety and insomnia for the past week. Her first symptoms appeared three years ago when she had 4 episodes of fugue.

\section{Results}

One year ago, with no obvious precipitating factor, she fainted in her garden and couldn't talk after recovering of that. At hospital, high blood pressure and expressive aphasia, were diagnosed at first but after full neurological examination and a cranial computed tomography, revealing no infarct, the patient was discharged as having 'conversion disorder' and mirtazapine and diazepam were prescribed. For the next weeks she didn't speak but communicated through writing. During her hospitalization in our ward of a psychiatric hospital it became obvious, through her writings, that the patient was psychotic (paranoid type), having persecutory delusions and also auditory hallucinations especially in the evening preventing her from sleeping. The diagnosis of psychosis was confirmed by an MMPI. EEG, CT scan and MRI were negative. She was treated with aripiprazole $15 \mathrm{mg}$. At first she started to whisper but was incomprehensible most of the time and her sleep improved. Three weeks later we could understand her 'whispering' and she was discharged. Nine months later she is functional, has no hallucinations or organized persecutory ideas. She still has some suspiciousness and though communicating very well is most of the time whispering!

\section{Conclusions}

Symptoms do not fully determine diagnosis or better to say the same symptoms can be found in many diagnostic categories. The severity and insistence of her 'typical' hysterical symptoms should have guided clinicians to the diagnosis of psychosis.

\footnotetext{
Author details

${ }^{1} 1$ st and 5th Department, Dromokaition Psychiatric Hospital, Athens, Greece.
}

${ }^{2} 5$ th Department, Psychiatric Hospital of Attica, Athens, Greece.

Published: 22 April 2010

\section{References}

1. Reed JL: The diagnosis of 'hysteria'. Psychol Med 1975, 5(1):13-7.

2. Linett LM: A case of pure word mutism: don't be fooled. Ann Emerg Med 1989, 18(11):1233-6.

3. Fisher W, Gebhardt M, Jahr H: Hysteria and psychosis. Psychiatr Neurol Med Psychol (Leipz) 1986, 38(6):324-30.

\section{doi:10.1186/1744-859X-9-S1-S133}

Cite this article as: Leotsakou et al.: Fugue and aphonia as first and foremost presentations of a case of psychosis. Annals of General Psychiatry 2010 9(Suppl 1):S133.

${ }^{1} 1$ st and 5th Department, Dromokaition Psychiatric Hospital, Athens, Greece 